\title{
The relationship of the immature platelet fraction with the diagnosis and severity of acute bronchiolitis in children.
}

\author{
MÜRT DOĞGN ${ }^{1}$, MEHMET KOSE ${ }^{1}$, Mehmet ÖZTÜRK ${ }^{1}$, melih hangul ${ }^{1}$, and \\ HÜMEYRA ASLANER ${ }^{1}$ \\ ${ }^{1}$ Erciyes University
}

December 20, 2020

\begin{abstract}
Objective: Acute bronchiolitis is one of the most common causes of hospitalization for children younger than 1 year. Although the prognosis for these children is generally good, the condition involves a risk of mortality. Here, we evaluate the immature platelet fraction (IPF) as a biomarker for the severity of acute bronchiolitis. Material and Methods: In total, 179 children who had been diagnosed with acute bronchiolitis were classified into three groups: mild, moderate, and severe bronchiolitis, and 80 healthy children were included as a control group. The diagnostic capacity of the IPF, mean platelet volume (MPV), platelet distribution width (PDW), white blood cell count (WBC), and platelet count (PLT) values to predict bronchiolitis was evaluated using receiver operating characteristic (ROC) curves and their respective areas under the curves (AUCs) calculated with $95 \%$ confidence intervals. Results: Patients with acute bronchiolitis had a larger IPF than their healthy counterparts ( $\mathrm{p}$ $<0.001)$. Additionally, a positive correlation was observed between the clinical severity of the disease and the IPF. The ROC curve analysis indicated that the IPF cut-off point for predicting acute bronchiolitis was $>3.2 \%$, with a sensitivity of $84 \%$ and specificity of $97 \%$. Our results clearly demonstrate that the AUCs for IPF, MPV, PDW, WBC, and PLT were statistically significant for the bronchiolitis versus the control group. The AUC was greatest for the IPF. Conclusion: The IPF is a new marker for diagnosing and evaluating the clinical severity of acute bronchiolitis.
\end{abstract}

\section{TÍTLE PAGE}

Manuscript Title: The relationship of the immature platelet fraction with the diagnosis and severity of acute bronchiolitis in children.

RunningTitle: Immature platelet fraction

Murat DOĞAN, MD

Medical Doctor

Department of Pediatrics Emergency, Erciyes University, Faculty of Medicine, Kayseri,Turkey

Mail Address: doktormurat033@hotmail.com

Phone: +905059025999

Business Number: +903522076666/ 25254

FaxNumber: +903524375825

ORCID: 0000-0003-2954-3845

Mehmet KÖSE, MD 


\section{Prof.Dr}

Department of Pediatrics Pulmonology, Erciyes University, Faculty of Medicine, Kayseri,Turkey Mail Addres:mhmtkose@yahoo.com

Phone: +905336496874

Business Number: $+903522076666 / 25468$

FaxNumber: +903524375825

ORCID: 0000-0002-3003-918X

Mehmet Adnan ÖZTÜRK, MD

Prof.Dr

Department of Pediatrics Emergency, Erciyes University, Faculty of Medicine, Kayseri,Turkey

Mail Address: adozturk2000@yahoo.com

Phone: +905334761166

Business Number: $+903522076666 / 25254$

FaxNumber: +903524375825

ORCID: 0000-0001-5397-1239

\section{Melih HANGÜL, MD}

Medical Doctor

Department of Pediatrics Pulmonology, Erciyes University, Faculty of Medicine, Kayseri,Turkey Mail Address:mhangul@gmail.com

Phone: +905053595233

Business Number: $+903522076666 / 25468$

FaxNumber: +903524375825

ORCID: 0000-0001-6226-0340

\section{Hümeyra ASLANER, MD}

Medical Doctor

Department of Family Physicians, Erciyes University, Faculty of Medicine, Kayseri,Turkey

Mail Address:drhumeyra@hotmail.com

Phone: +905066456944

Business Number: +903522076666

FaxNumber: +903524375825

ORCID: 0000-0002-3710-3893

Corresponding author and writer the first draft of the manuscript:

Murat DOĞAN, MD

Medical Doctor 
Department of Pediatrics Emergency, Erciyes University, Faculty of Medicine, Kayseri,Turkey

Mail Address: doktormurat033@hotmail.com

Phone: +905059025999

Business Number: +903522076666/25254

FaxNumber: +903524375825

ORCID: 0000-0003-2954-3845

Each author listed on the manuscript has seen and approved the submission of this version of the manuscript and takes full responsibility for the manuscript. This study was completed in accordance with the tenets of the Declaration of Helsinki. There is no conflict of interest. We didn't any support or sponsor in study design, the collection, analysis, and interpretation of data, the writing of the report and the decision to submit the paper for publication.Our study has not been presented before in any congress,symposium or meeting.

The relationship of the immature platelet fraction with the diagnosis and severity of acute bronchiolitis in children.

\section{Abstract}

Objective:Acute bronchiolitis is one of the most common causes of hospitalization for children younger than 1 year. Although the prognosis for these children is generally good, the condition involves a risk of mortality. Here, we evaluate the immature platelet fraction (IPF) as a biomarker for the severity of acute bronchiolitis.

Material and Methods: In total, 179 children who had been diagnosed with acute bronchiolitis were classified into three groups: mild, moderate, and severe bronchiolitis, and 80 healthy children were included as a control group. The diagnostic capacity of the IPF, mean platelet volume (MPV), platelet distribution width (PDW), white blood cell count (WBC), and platelet count (PLT) values to predict bronchiolitis was evaluated using receiver operating characteristic (ROC) curves and their respective areas under the curves (AUCs) calculated with $95 \%$ confidence intervals.

Results: Patients with acute bronchiolitis had a larger IPF than their healthy counterparts $(p<0.001)$. Additionally, a positive correlation was observed between the clinical severity of the disease and the IPF. The ROC curve analysis indicated that the IPF cut-off point for predicting acute bronchiolitis was $>3.2 \%$, with a sensitivity of $84 \%$ and specificity of $97 \%$. Our results clearly demonstrate that the AUCs for IPF, MPV, PDW, WBC, and PLT were statistically significant for the bronchiolitis versus the control group. The AUC was greatest for the IPF.

Conclusion: The IPF is a new marker for diagnosing and evaluating the clinical severity of acute bronchiolitis.

Keywords: Bronchiolitis, immature platelet fraction, inflammation

\section{1| INTRODUCTION}

Bronchiolitis is the most common lower respiratory tract infection in children younger than 1 year ${ }^{1}$. Viruses are the most common cause of acute bronchiolitis, especially respiratory syncytial virus. Bronchiolitis is characterized by airway obstruction and edema, increased mucus production, and the loss of airway epithelial cells. The clinical severity of bronchiolitis ranges from mild cases that can be treated on an outpatient basis to severe cases that require mechanical ventilation or extracorporeal membrane oxygenation in intensive care units ${ }^{2}$. The lack of any single biomarker for acute bronchiolitis that may be applied across all clinical scenarios is a recurring problem and has led many clinicians and researchers to pursue alternative diagnostic strategies. 
Platelets play an important role in hemostasis, inflammation, allergic reactions, and angiogenesis, as well as the repair and renewal of tissues. They also secrete mediators such as chemokines, cytokines, and coagulation factors, which provoke a strong inflammatory response and tissue regeneration. Platelet production in bone marrow increases and platelets are redistributed during the inflammatory response ${ }^{3}$. In addition to platelet count (PLT), platelet indexes include plateletcrit, mean platelet volume (MPV), platelet distribution width (PDW), and a new parameter called the immature platelet fraction (IPF). The IPF is the percentage of reticulated platelets that can be measured in the blood, and it may be used to quantify the production of bone marrow platelets. The IPF correlates directly with the thrombopoietic rate, increasing when platelet production rises and decreasing when production falls. Inflammation causes changes in the bone marrow, leading to increases in platelet production and the circulation of immature platelets ${ }^{4,5}$. Here, we evaluate the relationship between changes in platelet parameters such as IPF and the clinical severity of acute bronchiolitis.

\section{2 | MATERIAL AND METHODS}

A prospective study of children aged 1-24 months who were hospitalized with acute bronchiolitis was performed between December 2018 and May 2019. In accordance with American Academy of Pediatrics guidelines, a diagnosis of acute bronchiolitis was based on at least two of the following signs: chest retractions, tachypnea, and the first episode of wheezing or rales on auscultation following a viral upper respiratory tract infection in children aged younger than 24 months $^{6}$. The study included 179 patients with acute bronchiolitis and 80 healthy children. Inclusion criteria were: aged 1-24 months, first wheezing episode, no previous disease history, and no previous medication. Exclusion criteria were: chronic disease, premature birth, birth weight $<2500 \mathrm{~g}$, malnutrition, passive smoking, proven immune deficiency, proven or suspected acute bacterial infection, previous treatment with bronchodilators or corticosteroids, or having symptoms for more than 7 days. On admission, the clinical severity score (CSS) for acute bronchiolitis (i.e., a composite clinical score including respiratory rate, retraction, wheezing, and general condition) was used to evaluate patients, as previously described by Wang et al.$^{7}$ Each patient with bronchiolitis was classified into one of three groups, depending on whether they had mild, moderate, or severe bronchiolitis. Complete blood count measurements (including white blood cells, MPV, and platelets) were recorded from the blood samples taken on the first day of hospitalization using a BC-6800 analyzer (Mindray, Shenzhen, China). The IPF was recorded using flow cytometry and the reticulocyte/platelet channel of an XE-5000 automated hematology analyzer (Sysmex, Kobe, Japan) with a fluorescent dye containing polymethrin and oxazine. The IPF is the fraction (\%) of immature platelets in the total platelet population. No standard reference range for the IPF has been determined. Data from each patient recorded in the emergency room included: age, sex, disease history, medication, birth history, whether this was the first attack of bronchiolitis, weight, vital signs (i.e., heart rate, respiratory rate, tympanic temperature, and oxygen saturation when breathing ambient air, which was measured using pulse oximetry and expressed as $\mathrm{SpO}_{2}$ ). The control group included 80 healthy children who attended pediatric clinics for routine health checks or vaccinations. They had similar age/sex demographic characteristics to the children with bronchiolitis. Complete blood counts and IPF data were obtained from blood samples taken for routine testing of these children at their first visit. The families of all children were informed about the study objectives, and written informed consent was obtained before enrollment in the study. The study protocol was approved by the Ethics and Research Committee of Erciyes University (Kayseri, Turkey).

\subsection{Statistical analyses}

Statistical analyses were performed using SPSS software (ver. 21.0; IBM, Chicago, IL, USA). Continuous variables are expressed as medians (minimum-maximum) and categorical variables as values and percentages. Categorical data were compared using chi-square tests. For non-normally distributed data, the MannWhitney U test was used to determine whether or not differences between groups were statistically significant. Spearman's method was used to evaluate correlations between the IPF and other blood parameters if variables were not normally distributed. The most discriminating biomarkers for acute bronchiolitis were identified by drawing receiver operating characteristic (ROC) curves for each biomarker, and calculating sensitivity 
and specificity, as well as positive and negative predictive values. Areas under the curves (AUCs) for IPF, PLT, MPV, PDW, and white blood cell count (WBC) were evaluated for the acute bronchiolitis versus the control group. Logistic regression was used to identify associated factors and to calculate odds ratios and $95 \%$ confidence intervals. A $p$-value $<0.05$ was considered statistically significant.

\section{3 | RESULTS}

In total, 179 patients aged 1-24 months were included in this study, with a median age of 8 months (range, 1-24 months): $116(64.80 \%)$ of these patients were male and $63(35.20 \%)$ were female. The control group included 80 healthy children, also with a median age of 8 months (range, 2-24 months): 49 (61.2\%) of them were male and $31(38.8 \%)$ were female. The patient and control group did not differ significantly in age or sex $(p=0.619$ and $p=0.583$, respectively). Patients had significantly higher WBC, PLT, MPV, IPF, and PDW values compared with the control group $(p=0.000)$. The frequency of eosinophilia did not differ significantly between the patient and control groups $(p=0.756$; Table I).

The patient group included 48 (26.80\%), $104(58.10 \%)$, and $27(15.10 \%)$ children classified as having mild, moderate, and severe bronchiolitis, respectively. The median CSS was 6 (range, 2-12). No significant correlation was found between acute bronchiolitis CSS and age, sex, body temperature, oxygen saturation (\%), PLT, WBC, proportion of lymphocytes, or proportion of eosinophils $(p>0.05)$. The MPV value of the mild clinical severity group was significantly lower than those of the moderate $(p=0.002)$ and severe $(p=0.000)$ group, but MPV values did not differ significantly between the moderate and severe groups $(p$ $=0.142)$. IPF values differed significantly among the mild, moderate, and severe groups $(p<0.001)$. A positive correlation was observed between the CSS and the IPF $(p<0.001)$. The mild clinical severity group had a significantly lower PDW compared with the moderate and severe groups $(p=0.000)$, but the PDW values of the moderate and severe groups did not differ significantly.

ROC curve analyses were used to evaluate the performance of each biomarker in distinguishing acute bronchiolitis patients from controls, and in assessing acute bronchiolitis severity. The AUC for IPF, MPV, PDW, WBC, and PLT was $0.95,0.85,0.58,0.79$, and 0.71 , respectively. ROC curve analysis suggested that the cut-off for using IPF to predict bronchiolitis was $>3.2$, with a sensitivity of $84 \%$ and a specificity of $97 \%$. The positive and negative predictive values of the IPF were $98.7 \%$ and $73.5 \%$, respectively. The AUC was greatest for IPF (Figure1). IPF, MPV, and PDW ROC curves showed significant sensitivity and selectivity in patients $(p<0.001$; Table III). For all patients, this was the first bronchiolitis attack. At admission, the median body temperature of patients was $37^{\circ} \mathrm{C}\left(36-39.3^{\circ} \mathrm{C}\right)$ and their median oxygen saturation was $91 \%$ (75-99\%). In total, 99 patients had a chest X-ray finding (e.g., diffuse interstitial consolidation or increased aeration), 68 patients were given antibiotics, and 71 patients received steroid therapy (all patients in the severe group and $42.3 \%$ of patients in the moderate group). All patients were provided with supportive therapy such as hydration, oxygen, and salbutamol nebules if indicated.

\section{4 | DISCUSSION}

We found that the IPF, which is a new platelet index, was greater in patients with acute bronchiolitis than in healthy individuals and was positively correlated with the clinical severity of acute bronchiolitis. To our knowledge, this is the first study to investigate the relationship between acute bronchiolitis and IPF. Some cytokines and mediators are activated as part of the response to inflammatory conditions. Inflammation mediates the activation of endotoxins and cytokines, causing immature reticulated platelets (i.e., IPF) to be transferred from the bone marrow to peripheral blood. Platelets stimulate receptors as part of the inflammatory response to pathogens, and interleukins are released to suppress inflammation ${ }^{8-10}$. Therefore, the IPF is a biochemical and hematological systemic inflammatory marker that can function as an index of platelet activation and production rate in the bone marrow ${ }^{11}$. Diagnostic accuracy studies performed over the last few years suggest that IPF levels can provide clinically relevant information regarding inflammatory activity and disease prognoses ${ }^{4,11,12}$. Roberto et al . reported that IPF levels increased in patients with sepsis before sepsis was observed clinically, and they also found that IPF was a better biomarker for sepsis than procalcitonin or C-reactive protein ${ }^{13}$. Park et al . found that IPF levels were significantly higher in patients 
with sepsis than in individuals who did not have sepsis ${ }^{5}$. Rodolfo et al . evaluated the IPF as a biomarker for sepsis diagnosis and severity and found that it was correlated with sepsis severity scores and had the highest diagnostic accuracy for sepsis among all the clinical and laboratory parameters assessed ${ }^{14}$. Another study found that IPF was strongly positively correlated with MPV and PDW, and attributed this to an increase in the number of larger and wider platelets that appeared after the destruction of pro-inflammatory cytokines and endotoxins as part of a severe inflammatory response ${ }^{15}$. Together, these findings suggest that IPF levels increase in inflammatory diseases such as sepsis and may be used as an indicator of the inflammatory response. Our observation that the IPF was greater in patients with acute bronchiolitis than in healthy individuals is consistent with previous studies. The clinical severity of acute bronchiolitis was positively correlated with the IPF. Therefore, patients with acute bronchiolitis and high IPF values should be monitored closely after admission to the emergency department. IPF values higher than $3.2 \%$ had a specificity of $97 \%$ and a sensitivity of $84 \%$ for clinical deterioration of acute bronchiolitis.

Platelets play an important role in inflammatory responses, and the PLT increases in many inflammatory diseases. Studies have found that patients with acute bronchiolitis have higher PLTs than healthy individuals ${ }^{16,17}$. Our patient group had significantly greater PLT values than our healthy control group, and this observation is consistent with previous studies. Our severe bronchiolitis group had higher PLT values compared to our mild bronchiolitis group, but the difference was not statistically significant. During an inflammatory response, depolymerization occurs in the microtubular structure of platelets, and changes also occur in the structure of actin that becomes polymerized; consequently, platelets change their shapes and these changes are reflected in the MPV ${ }^{18}$. Changes in MPV can be observed before changes in platelet number, so changes in MPV may be useful for predicting inflammation at an early stage and determining its subsequent severity ${ }^{19-21}$. Among our study population, the MPV was significantly higher in those with moderate and severe bronchiolitis than in those with mild bronchiolitis or in healthy controls, so MPV values may be used to estimate prognoses and the severity of acute bronchiolitis; patients with an MPV value greater than $9 \mathrm{fL}$ should be monitored carefully for clinical deterioration. Several previous studies have evaluated the relationship between acute bronchiolitis and MPV. Gökçe et al . ${ }^{22}$ found that patients with acute bronchiolitis had higher MPVs than their healthy counterparts, but found no statistically significant differences in MPVs between different bronchiolitis groups. Higher MPVs were observed in children with influenza A respiratory tract infections than in healthy children ${ }^{23}$. MPVs were also found to be higher in children with pneumonia, asthma, or sepsis than in healthy children ${ }^{24-26}$. Gasparyan et al.$^{27}$ found that MPV values were increased in patients with mild inflammation, and they attributed this finding to the transfer of large activated platelets to the site of infection as the severity of inflammation increased. Together, these results suggest that the MPV may also be used to estimate prognoses and the severity of other inflammatory diseases.

PDW, another platelet volume index, shows the variation in platelet diameters and varies by platelet activation. An increase in PDW may occur as a result of swelling, disruption, or platelet immaturity ${ }^{20}$. In our study, PDW values were significantly greater in patients with moderate and severe bronchiolitis cases than in those with mild bronchiolitis or healthy controls. Ergulet al . found greater PDW values in children with acute bronchiolitis than in healthy children ${ }^{17}$. PDW may also be greater in patients with sepsis than in healthy individuals, and may be used as a prognostic indicator for patients with sepsis ${ }^{26,28}$.

The present study had some limitations. It was not a multi-center study. Additionally, we did not characterize the viruses that cause acute bronchiolitis and compare the corresponding clinical severities. The IPF is a novel indicator of inflammation, and it is also a new marker for diagnosing and evaluating the clinical severity of acute bronchiolitis. Larger prospective studies are needed to clarify the clinical significance of using IPF values to assess patients with acute bronchiolitis.

\section{ACKNOWLEDGMENTS}

This study received no specific grant from any funding agency in the public, commercial, or not for profit sectors.

CONFLICT OF INTERESTS 
The author declares that there are no conflict of interests

\section{AUTHOR CONTRIBUTIONS}

Concept and design: MD, MK, MAÖ, MH. Acquisition, analysis, or interpretation of data: MD, HA, MH. Drafting of the manuscript: MAÖ, MK, MD, MH. Critical revision of the manuscript for important intellectual content: MAÖ, MK, HA, MD. Statistical analysis: MD,HA. Administrative, technical, or material support: MAÖ, MK, MD. Supervision: MD, MK, MH.

\section{REFERENCES}

1. Fleming PF, Richard S, Waterman K, Davis PG, Kamlin CO, Stewart M, Sokol J. Medical retrieval and needs of infants with bronchiolitis: an analysis by gestational age. J Paediatr Child Health 2013;49:E227-31.

2. Wright M, Piedimonte G. Respiratory syncytial virus prevention and therapy: past, present, and future. Pediatr Pulmonol 2011; 46(4):324-47. [PubMed: 21438168]

3. Morrell CN, Aggrey AA, Chapman LM, Modjeski KL. Emerging roles for platelets as immune and inflammatory cells. Blood 2014;123:2759 -2767.

4. Ault KA, Rinder HM, Mitchell J, Carmody MB, Vary CP, Hillman RS. The significance of platelets with increased RNA content (reticulated platelets): a measure of the rate of thrombopoiesis. Am J Clin Pathol 1992; 98:637-646

5. Park S H, Ha S O, Cho Y U, Park C J, Jang S, Hong S B. Immature platelet fraction in septic patients: clinical relevance of immature platelet fraction is limited to the sensitive and accurate discriminationof septic patients from non-septic patients, not to the discrimination of sepsis severity. Ann Lab Med 2016;36:1-8.

6. American Academy of Pediatrics Subcommittee on Diagnosis and Management of Bronchiolitis. Diagnosis and management of bronchiolitis. Pediatrics 2006;118:1774 - 93.

7.Wang EE, Milner RA, Navas L, Maj H. Observer agreement for respiratory signs and oximetry in infants hospitalized with lower respiratory infections. Am Rev Resp Dis. 1992;145:106 -15.

8. Wiersinga WJ, Leopold SJ, Cranendonk DR, van der Poll T. Hostinnate immune responses to sepsis. Virulence 2014; 5: 36-44, doi:10.4161/viru.25436 (2014).

9. Lay MK, Gonzalez PA, Leon MA, Cespedes PF, Bueno SM, Riedel CA, Kalergis AM. Advances in understanding respiratory syncytial virus infection in airway epithelial cells and consequential effects on the immune response. Microbes Infect 2013;15(3):230-42. [PubMed: 23246463]

10. Franco AT, Corken A, Ware J. Platelets at the interface of thrombosis, inflammation, and cancer. Blood $2015 ; 126(5): 582-8$.

11. Jiang LB, Ma YF, Zhang M. Can mean platelet volume predict the prognosis of patients with acute kidney injury requiring continuous renal replacement therapy? J Crit Care 2015;30:207.

12. Morrell CN, Aggrey AA, Chapman LM, and Modjeski KL. Emerging roles for platelets as immune and inflammatory cells. Blood 2014; 123, 2759-2767, doi:10.1182/blood-2013-11-462432.

13. De Blasi RA, Cardelli P, Costante A, Sandri M, Mercieri M, Arcioni R. Immature platelet fraction in predicting sepsis in critically ill patients. Intensive care medicine 2013; 201339:636-643, doi: 10.1007/s00134012-2725-7.

14. Enz Hubert RM, Rodrigues MV, Andreguetto BD, M Santos T, et al. Association of the immature platelet fraction with sepsis diagnosis and severity.Sci Rep 2015 Jan 26;5:8019.

15. Djuang M H, Ginting F, Hariman H. Immature platelet fraction in bacterial sepsis severity assessment. IOP Conf. Series: Earth and Environmental Science 2018; 125:012024

16. Yeaman MR. Platelets: at the nexus of antimicrobial defence. Nat Rev Microbiol 2014; 12: 426-37. 
17. Ergul A B, Torun YA, Uytun S, Aslaner H, Kısaaslan AP, Şerbetçi MC. Reduction in mean platelet volume in children with acute bronchiolitis Turk Pediatri Ars 2016; 51: 40-5.

18. Flad HD, Brandt E. Platelet-derived chemokines: pathophysiology and therapeutic aspects. Cell Mol Life Sci 2010; 67: 2363-86.

19. Andrews RK, Berndt MC. Platelet physiology and thrombosis. Thromb Res 2004; 114: 447-53.

20. Bessman JD, Gilmer PR, Gardner FH. Use of mean platelet volume improves detection of platelet disorders. Blood Cells 1985; 11: 127-35.

21. Kisacik B, Tufan A, Kalyoncu U, Karadag O, Akdogan A, Ozturk MA, Kiraz S, Ertenli I, Calguneri M. Mean platelet volume (MPV) as an inflammatory marker in ankylosing spondylitis and rheumatoid arthritis. Joint Bone Spine 2008; 75: 291-4.

22. Gökce Ş, Kurugöl Z, Suner A. The Role of Mean Platelet Volume in the Early Detection o f Acute Bronchiolitis: A Prospective Study. Clin Respir J 2018 Oct;12(10):2513-2518.

23. Fei Y, Zhang H, Zhang C. The application of lymphocyte* platelet and mean platelet volume/platelet ratio in influenza A infection in children. J Clin Lab Anal 2019;33:e22995.

24. Karadag-Oncel E, Ozsurekci Y, Kara A, Karahan S, Cengiz AB, Ceyhan M. The value of mean platelet volume in the determination of community acquired pneumonia in children. Ital J Pediatr 2013; 39: 16.

25. Dogru M, Aktas A, Ozturkmen S. Mean platelet volume increased in children with asthma. Pediatr Allergy Immunol 2015 Mar 26. doi: 10.1111/pai.12381. [Epub ahead of print

26. Guclu E, Durmaz Y, Karabay O. Effect of severe sepsis on platelet count and their indices. Afr Health Sci 2013; 13: 333-8.

27. Gasparyan AY, Ayvazyan L, Mikhailidis DP, Kitas GD. Mean platelet volume: a link between thrombosis and inflammation? Curr Pharm Des 2011; 17: 47-58.

28. Akarsu S, Taskin E, Kilic M, Ozdiller S, Gurgoze MK,Yılmaz E, Aygun AD. The effects of different infectious organisms on platelet counts and platelet indices in neonates with sepsis: is there an organismspecific response? J Trop Pediatr 2005; 51: 388-91.

\section{Hosted file}

TABLES.pdf available at https://authorea.com/users/383886/articles/499709-therelationship-of-the-immature-platelet-fraction-with-the-diagnosis-and-severity-of-acutebronchiolitis-in-children

\section{Hosted file}

F\selectlanguage\{polish\}İ \selectlanguage\{english\}GURE.pdf available at https://authorea.com/ users/383886/articles/499709-the-relationship-of-the-immature-platelet-fraction-withthe-diagnosis-and-severity-of-acute-bronchiolitis-in-children 